der Ränder beider Planeten um diese Zeit $10^{\prime \prime 2}$

d. h. $\frac{1}{3}$ des scheinb. Durchmessers des Jupiter betragen.

Für Berlin ist die Rechnung zum Theil unter Berücksichtigung der Parallaxe wiederholt worden und es hat sich hiernach ergeben:

$\begin{array}{cc}\text { m.Zt. } & \mathcal{s} \\ \mathbf{1 6}^{\text {b } 35^{\mathrm{m}}} & \mathbf{3 3}^{\prime \prime} 4 \\ \mathbf{3 6} & 33,3 \\ \mathbf{3 7} & 33,3 \\ 38 & 33,4 \\ 39 & 33,5 \\ 40 & 33,5\end{array}$

Für Berliu wird daher der kleinste Abstand, nämlich $33^{\text {" } 3}$

um $16^{\mathrm{h}} 36^{\mathrm{m}} 5 \mathrm{~m}$. B.Z. eintreten, die Rănder werden um $12^{\mu \prime} 9$

von einander entfernt erscheinen.

Die in den letzten Bänden der Astronom. Nachrichten veröffentlichten Königsberger Beobachtungen beider Planeten machen es wahrscheinlich, dass die nach den Tafeln be- rechnete Decl. $\%$ um etwa 9", die Decl.4 nm $4^{\mu}$ vermindert werden muss. Hiernach dürfte der kleinste Abstand um $5^{\text {" }}$ grösser ausfallen.

Dem unbewaffineten Auge werden zur Zeit der kleinsten Entfernung beide Planeten als e in Gestirn erscheinen, allein in Berlin wird dieses Zeitmoment erst nach Sonnen-Aufgang stattinden, indem an diesem Tage

$$
\begin{aligned}
& + \text { um } 14^{\mathrm{b}} 27^{\mathrm{m}} \\
& 4=1436 \\
& \odot=163
\end{aligned}
$$

aufgehen. Der kleinste Abstand wird daber um $34^{\mathrm{m}}$ nach Sonnen-Aufgang in Berlin eintreten und man wird sich vach einem $8-10^{\circ}$ gegen Westen von Berlin liegenden Orte begeben müssen, um diese Erscheinung vor Aufgang der Sonne zu sehen.

Bein Aufgange des Jupiter in Berlin wird der kleinste Abstand der Mittelpunkte $5^{\prime} 4^{\prime \prime} 0$, beim Aufgange der Sonne 1'30" 2 betragen.

Berlin 1858 Sept. 19.

\title{
Elemente und Ephemeride der Pomona, von Herrn Lesser.
}

Bei der folgenden Bahnbestimnung kounte ich 3 Oppositionen benutzen. Die Beobachtungen derselben vereinigte ich zu 6 Normalortern, nämlich: mittl. Berl. Zt.

1. 1854 Nov. 11,0
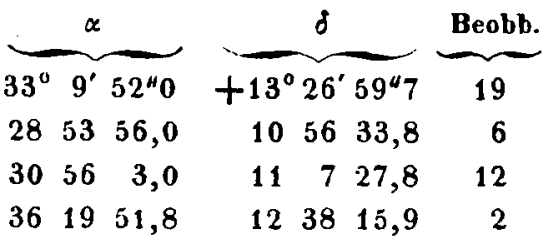

III. $1855^{\circ}$ Jan 14,0

$33^{\circ} 9^{\prime} 52^{4} 0$

$28 \quad 53 \quad 56,0$

$\begin{array}{llll}30 & 56 & 3,0\end{array}$

$1238 \quad 15,9$

14

Y. 1856 Mä 3,0

$\begin{array}{lll}36 & 19 & 51,8\end{array}$

$+53058,6$

19

Vl. 1857 Juli 15,0

$280 \quad 34 \quad 32,2$

$\begin{array}{lll}-14 & 59 & 40,1\end{array}$

19

Um un die Beobachtungen genügend darzustellen, ging ich von Elenenten aus, welche auf 3 Normalörtern der $1^{\text {sten }}$ und $2^{\text {ten }}$ Erscheinung beruhen; diese Elemente sind:
I.

1855 Januar 5,0 mittl. Berl. Zt.

$$
\begin{aligned}
& L=57^{\circ} 38^{\prime} 18^{\prime \prime} 4 \\
& M=223 \quad 7 \quad 5,5
\end{aligned}
$$

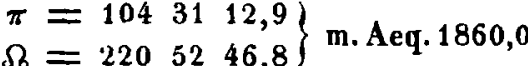

$$
\begin{aligned}
& i=5293,6 \\
& \varphi=44327,6 \\
& \log a=0,412705 \\
& =\mu=2,930950
\end{aligned}
$$

Mit diesen Elementen berechnete ich die Störungen durch Jupiter und Saturn von Neuem und entwickelte für die Örter I. III. V. und VI. folgende Bedingungs-Gleichungen :

$$
\begin{aligned}
& 0=+0 " 3+1,2854 x+2,0363 y-1,1502 z-0,8991 u-0,4238 v-0,00398 w \\
& 0=+2,0+0,8960=+1,3725=-0,9879=-0,5284=-0,3129=+0,06239= \\
& 0=-2,1+1,7499=-1,9703=-2,3672=+7,0172=-0,2416=-0,46621= \\
& 0=+107,3+1,6340=-0,2207=+3,2347=+14,8300=+0,0077=-0,17995= \\
& 0=-0,1+0,31898=+0,5098=-0,27609=-0,2418=+1,4106=+0,01596= \\
& 0=+1,4+0,21648=+0,3579=-0,20723=-0,2042=+1,0136=-0,24084= \\
& 0=-0,6-0,57699=+0,7004=+0,69827=-2,4688-0,5086=-1,46530= \\
& 0=+17,4+0,20167=+0,0518=+0,41362=+1,9848=-0,6737=+1,45855= \\
& \text { Hier ist } x=\Delta L, y=\mathrm{I}^{1} \sigma \pi, z=\Delta \varphi, u=100 \Delta \mu, v=\frac{1}{5} \Delta \Omega, w=\Delta i \text {. }
\end{aligned}
$$

Bei der weiteren Bearbeitung nahn ich für alle Gleichungen dasselbe Gewicht an, da den beautzten Normalörtern nabe gleich viele Beobachtungen zu Grunde liegen. Die Auflösung der Gleichungen ergab als wahrscheinlichste Werthe: 


$$
\begin{array}{lll}
x=+38^{\prime \prime} 853 & \text { oder } & \Delta L=+0^{\prime} 38^{\prime \prime} 85 \\
y=-25,963 & \Delta \pi=-419,63 \\
z=+8,693 & \Delta \varphi=+08,69 \\
u=-13,803 & \Delta \mu=-0,1380 \\
v=-0,590 & \Delta \Omega=-5,90 \\
w=-0,371 & \Delta i=-0,37
\end{array}
$$

Die verbesserten Elemente werden demnach:

\section{II.}

1855 Januar 5,0 mittl. Berl. Zt.

$$
\begin{aligned}
& M=223^{\circ} 12^{\prime} 3^{\prime \prime} 98
\end{aligned}
$$

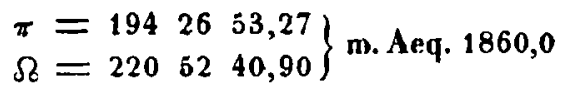

$$
\begin{aligned}
& i=5293,23 \\
& \varphi=44336,29 \\
& \log a=0,4127512 \\
& =\mu=2,9308797
\end{aligned}
$$

Die Daratellung der Beobachtungen ist genügend; man erhält nämlich als Febler:

$$
\begin{aligned}
& \text { Bedingungogleich. Directe Berechn. }
\end{aligned}
$$

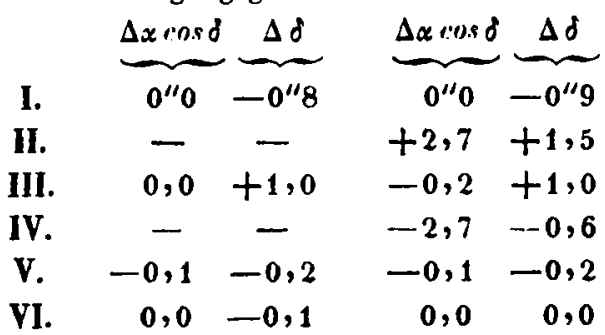

Die Gewichte der einzelnen Verbesserungen sind jedocb im Allgemeinen sehr klein und es kann daher eine Abweichung in der bevorstehenden Opposition nicht befremden. Ich halte es für überflüssig, die mittleren Fehler anzufuhren, da diese bei der geringen Anzahl der Bedingungsgleichungen nur wenig Werth haben können. Die Länge des Perihels hleibt besonders unsicher.
Aus den Elementen 11. habe ich nun, unter Berücksichtigung der Störungen, nachstehende Ephemeride abgeleitet.

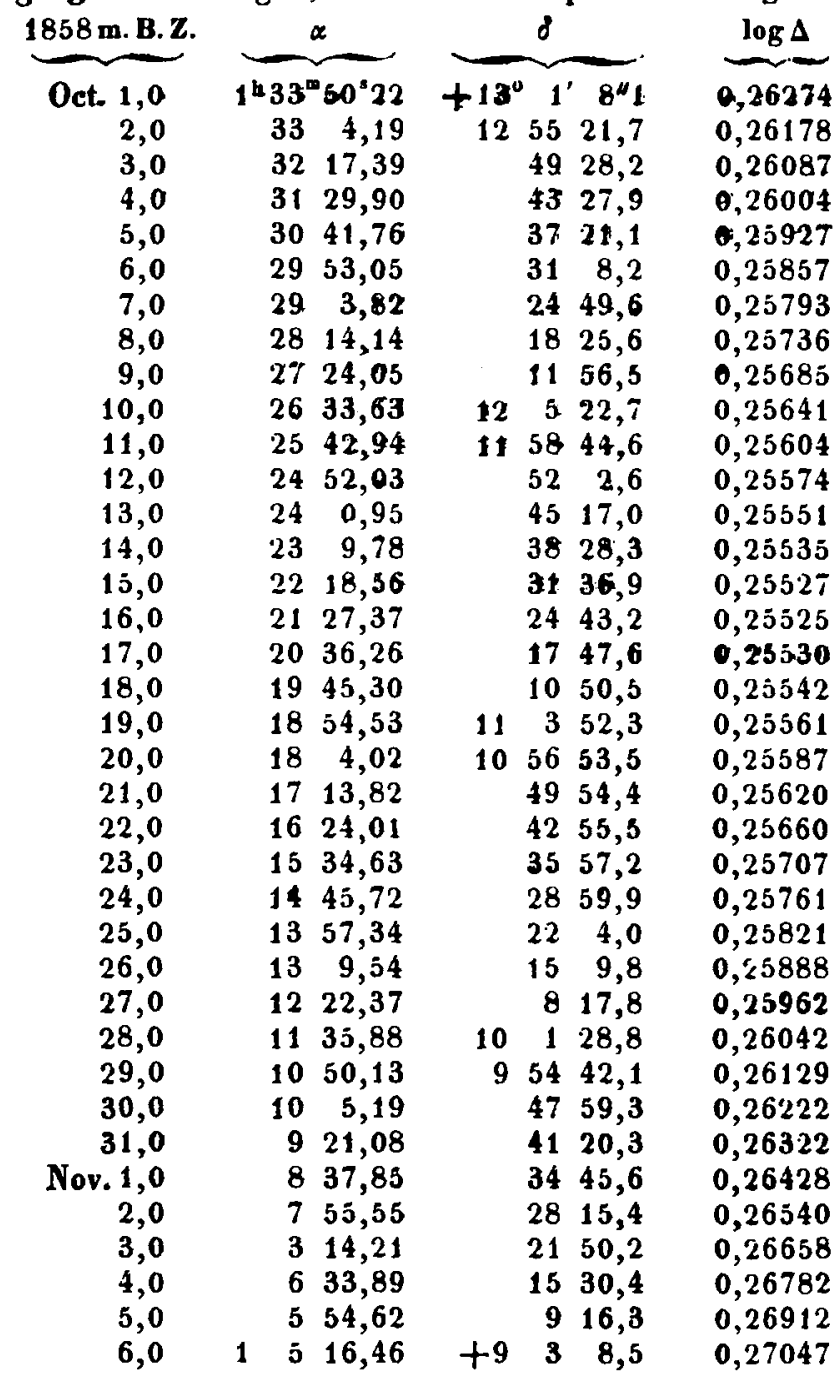

Opposition 1858 Octb. $165^{\text {h }} 21^{\mathbf{m}} 5$. Lichtstärke $=0,665$. Grösse $=11,4$.

Berlin 1858 Sept. 13.
O. Lesser.

\section{Elemente und Ephemeride des Donati'schen Cometen, von Herrn Löxy.}

Das folgende neue Elementensystem des Donati'schen

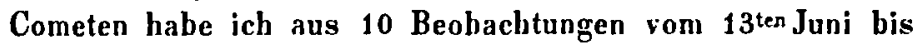
$14^{\text {ter }}$ Septemher abgeleitet. Die Bahn geht durch die äussersten Orte u. die übrigbleibenden Fehler der beiden Zwischenorte, welche einen etwas grösseren Betrag hahen, als dies sonst gewöhnlich bei guten Beobachtungen vorkoummt, erlauben doch noch keinen bestimmten Scbluss über die eigent- liche Natur der Bewegung. Ich werde sohald die Umstände sich günstiger gestalten, eine genauere Rechnung folgen lassen. Die hüchst eigenthümliche Bewegung dieses Cometen veranlasste mich schon früher zu einer wiederholten Rechnung. lch benutzte dazu 19 Beobachlungen vom 9 ten Juni bis $17^{\text {ten }}$ August. Das Erscheinen der Bralns'schen Elemeute und Ephemeride, die übereinstimmend mit den meinigen waren, 